\title{
Conjugate heat transfer through nano scale porous media to optimize vacuum insulation panels with lattice Boltzmann methods
}

\author{
Jesse Ross-Jones a,b,c,*, Maximilian Gaedtke ${ }^{\mathrm{b}, \mathrm{c}}$, Sebastian Sonnick ${ }^{\mathrm{a}, \mathrm{b}}$, \\ Matthias Rädle ${ }^{\mathrm{a}}$, Hermann Nirschl ${ }^{\mathrm{b}}$, Mathias J. Krause ${ }^{\mathrm{b}, \mathrm{c}}$ \\ ${ }^{a}$ CeMOS (Center of Mass Spectrometry and Optical Spectroscopy), Mannheim University of Applied Sciences, Mannheim, Germany \\ ${ }^{\mathrm{b}}$ Institute for Mechanical Process Engineering and Mechanics, Karlsruhe Institute of Technology, Karlsruhe, Germany \\ ' Lattice Boltzmann Research Group, Karlsruhe Institute of Technology, Karlsruhe, Germany
}

Keywords:

Rarefied gas dynamics

Nano-porous materials

Lattice Boltzmann method

Vacuum insulation

Mesoscopic methods

Nano-porous silica

\section{A B S T R A C T}

Due to reduced thermal conductivity, vacuum insulation panels (VIPs) provide significant thermal insulation performance. Our novel vacuum panels operate at reduced pressure and are filled with a powder of precipitated silicic acid to further hinder convection and provide static stability against atmospheric pressure. To obtain an in depth understanding of heat transfer mechanisms, their interactions and their dependencies inside VIPs, detailed microscale simulations are conducted.

Particle characteristics for silica are used with a discrete element method (DEM) simulation, using open source software Yade-DEM, to generate a periodic compressed packing of precipitated silicic acid particles. This aggregate packing is then imported into Open $L B$ (openlb.net) as a fully resolved geometry, and used to study the effects on heat transfer at the microscale. A three dimensional Lattice Boltzmann method (LBM) for conjugated heat transfer is implemented with open source software OpenLB, which is extended to include radiative heat transport. The infrared intensity distribution is solved and coupled with the temperature through the emissivity, absorption and scattering of the studied media using the radiative transfer equation by means of LBM. This new holistic approach provides a distinct advantage over similar porous media approaches by providing direct control and tuning of particle packing characteristics such as aggregate size, shape and pore size distributions and studying their influence directly on conduction and radiation independently. Our aim is to generate one holistic tool which can be used to generate silica geometry and then simulate automatically the thermal conductivity through the generated geometry.

\section{Introduction}

Energy efficiency is currently a very active topic of discussion and concern [1]. Buildings, homes and transport vehicles require energy and insulation to keep their occupants and/or wares at acceptable temperatures. Vacuum insulation panels (VIPs) are of interest to industry sectors looking to improve thermal efficiency [1]. A VIP provides significant thermal

\footnotetext{
* Corresponding author at: CeMOS (Center of Mass Spectrometry and Optical Spectroscopy), Mannheim University of Applied Sciences, Mannheim, Germany.

E-mail address: j.ross-jones@hs-mannheim.de (J. Ross-Jones).
} 
Table 1

Comparison of thermal conductivity $\lambda$ of high performance thermal insulation materials [3] (EPS and XPS are expanded and extruded polystyrene respectively).

\begin{tabular}{lll}
\hline Material & Thermal conductivity in $\mathrm{W} / \mathrm{mK}$ & Density in $\mathrm{kg} / \mathrm{m}^{3}$ \\
\hline Polystyrene & $0.034-0.036$ & $15-30$ \\
(EPS, XPS) & $0.033-0.040$ & $8-500$ \\
Mineralwool & $0.004-0.008$ & $150-350$ \\
VIP &
\end{tabular}

insulation performance $(0.004-0.008 \mathrm{~W} /(\mathrm{mK}))$ [2]. Due to this very low thermal conductivity, space required by insulation can decrease 5 to 10 times (see Table 1) when compared to using traditional thermal insulation materials. VIPs have already found widespread use in stationary refrigeration equipment [2]. Limited adoption, in house and building construction, has been seen due to cost, limited lifetime and risk of damage during installation and subsequent use of the panel. However, by increasing the internal pressure of the VIP while maintaining a low effective thermal conductivity, not only can the lifetime of the panel be extended, the increased thermal conductivity of a damaged panel as well as the cost of production would decrease.

To increase the minimum required pressure inside the VIP, the geometry of the packing medium can be modified. In order to understand effects of different packing geometries such as porosity and aggregate size on the individual contributions that make up the heat transfer through the VIP, a high fidelity model of the packing geometry is required.

Previous work is available which study heat transfer through VIPs. These works are divided here into two groups based on the form in which the geometry used for the simulation is generated. The first method generates the geometry based on idealized elementary units or fractal geometry, the second based on inhomogeneous procedurally generated geometry. The advantage of geometry based on elementary units lies in the relative simplicity of the mathematical formulation. For example, Enguehard et al. [4] use transmission electron microscopy (TEM) images of nanoporous silica as a basis in order to generate elementary bricks which emulate porous properties. Similarly, Spagnol et al. [5] present a method for modeling nanoporous silica with fractal geometry. Knowing the fractal dimension of the aggregates and agglomerates, the geometry is modeled with procedurally generated models of the porous structure. In another work, Jae-Sung Kwon [6] presented various methods for modeling VIPs as idealized geometry, with a focus on modeling the solid and gaseous thermal conductivity as well as radiation. The paper discusses modeling the contact area of different packing materials and compares these values to values reported by Fricke et al. [7]. Extending upon the models of Jae-Sung Kwon [6], Kim et al. [8] used the idealized geometry to study the effects of conductivity and radiation. The results of the models are compared to measurements. Rochais et al. [9] also present a method for procedural generation of VIP nanostructure geometry based primarily on the fractal dimension and repetitions of periodic base structures (square-shaped, diamond-shaped, brick-shaped). The geometry is then used to simulate effects of diffusion and conduction. Coquard et al. [10] also look into modeling the thermal conductivity of VIPs while also considering the effects of moisture. The geometry is modeled in the form of elementary cubes with a particular porosity and contact surface area.

While it is possible to closely approximate the geometry using the aforementioned methods, it is not possible to make changes to the geometry once generated, nor is it possible to produce inhomogeneous geometries. Furthermore, manual involvement is usually needed to transfer the generated geometry to the CFD simulation. Lallich et al. [11] present a method which uses a diffusion limited cluster-cluster aggregation algorithm, which is used to model silica aggregates with a fractal dimension between 1.8 and 2.5. The study is limited to studying radiation transfer in a vacuum. The use of lattice Boltzmann methods (LBM) to study heat transfer (conduction and radiation) through complex porous (foam) structures is presented by Wang et al. [12]. Similarly, Ferkl et al. [13] presented a method for 1D simulation of porous foam media for simulation of heat transfer with some extensions to 3D. Both these works focus on foam structures and are not able to simulate aggregate structures.

Wang et al. [14] presented a geometry generation algorithm to simulate the formation of porous media, based on four parameters, core distribution probability, growth directionality, porosity and phase interaction growth probability. While this method can model many different types of porous media, it does not facilitate modification of the geometry, e.g. compression. Once the geometry is generated, LBM is used to simulate conduction through the media, radiation was assumed to be negligible. However, radiation becomes a major component of the thermal conductivity and at low pressures can contribute around $40 \%$ of the heat flux [15]. Additionally, Qu et al. [16] present a method for simulating heat transfer in Silica Aerogels with LBM and compare their simulation results to published literature values. Using stochastically generated porous geometry, heat transfer due to gas and solid heat conduction with radiation is simulated. While this method allows direct control over pore size and overall porosity of the generated geometry, the method is implemented only in two dimensions. Falcucci et al. have investigated the use of LBM in the transition Knusden regime $(0.1>\mathrm{Kn}>1)$ and introduce a 'sputtering' boundary condition which redistributes outgoing lattice velocities to fit a probability distribution [17]. The authors were able to show fair agreement between their results and theoretical/experimental values for complex nanoporous geometries [18,19]. In the present study, convection is assumed to be negligible when the pore size is smaller than $3 \mathrm{~mm}$ [20] and an average Knudsen number is computed at the start of each simulation and used for the whole domain. 
The advantage of LBM is, on the one hand, the use of simple grids in combination with an efficient parallel algorithm, which allows resolving complex geometries with greater ease than with comparable methods. On the other hand, no special interface consideration is needed in LBM to assure temperature and heat flux continuities at the solid-fluid interfaces.

In this work a method for procedurally generating a compressed packing of 3D spherical silica and thereafter simulating the effective heat transfer through VIPs without manual intervention, is presented. The three dimensional geometry generation method implemented provides a distinct advantage over other porous media approaches by allowing direct control and tuning of particle packing characteristics such as aggregate size, shape and pore size distributions and studying their influence directly on conduction and radiation independently. Radiation and conduction are coupled in order to simulate heat transfer at the nanoscale and for a range of pressures, from 1 bar down to $1 \mathrm{mBar}$. This enables an in depth understanding of the heat transfer mechanism at the nanoscale and leads to both an improvement in the lifetime and reduction of the manufacturing costs of VIPs. Simulation results are compared to measurements taken of VIPs, produced with precipitated silica and pre-compressed under different pressures, as well as to literature values for silica VIPs. OpenLB is used as it is an open source implementation of LBM with a parallel execution capable of handling complex geometry [21]. Our aim is to generate one holistic tool which can be used to generate silica geometry, and then automatically simulate the thermal conductivity through the generated geometry. The simulations will be performed over a range of internal pressures and the geometry compressed over varying degrees. We will therefore be able to increase the minimum pressure required for a low effective heat transfer, which yields both an improvement in the lifetime and reduction of the manufacturing costs of VIPs.

This work begins by presenting the different thermal transfer LBM components simulated in VIPs, including the effects of low pressure and small pore size. Subsequently, the method used to generate the silica packing geometry is introduced. Following, the measurement system used to measure the thermal conductivity of the VIPs is presented. Next, convergence of the simulation results as a function of resolution is discussed. Lastly, the simulation results are compared to measured thermal conductivity of VIPs.

\section{Methods}

Our holistic approach is composed of two parts, the first generates the silica particle geometry with YADE, the second simulates the effective thermal conductivity through the geometry using OpenLB. We follow these two sections by describing the physical measurements of thermal conductivity through VIPs which are used to compare against the simulation results.

\subsection{Geometry generation approach}

Yet Another Dynamic Engine (YADE DEM) is used to generate the geometry and compress the resulting aggregates [22]. The process is divided into three steps, generating the aggregates, generating a loose packing of said aggregates and lastly compressing the packing. This geometry is passed automatically to OpenLB to simulate the effective thermal conductivity as described in Section 2.2 .

The first step uses the YADE function makeCloud, which creates a random loose packing of spheres inside a parallelepiped. Parameters for particle size distribution, aggregate size, and aggregate shape are provided to makeCloud to create a set of aggregates. Each aggregate is generated with different random seed values so as to create a randomized set of aggregates. These aggregates are saved as individual text files defining particle locations and radii.

In the second step the YADE function makeClumpCloud is used with parameters defining an initial bounding box and the set of aggregate to be used. The function makeClumpCloud creates a loose periodic packing of aggregates chosen equally from the input set to fill the volume. This volume is then saved to a text file defining the particle locations, radii as well as an index defining to which aggregate the particle belongs.

Lastly, in step 3, the volume in step 2 is compressed periodically on all three axes using the YADE compression engine PeriTriaxController, shown in Fig. 1 (The YADE compression engine produces compacted geometry and should not be confused with gas compressibility). Of note is that the particles within an aggregate are not internally compressed, only the aggregates themselves to one another. A maximum strain can be defined as a stopping criterion for the compression engine. Once the compression is completed, the geometry is exported to an XML file containing particle locations and radii. This geometry can then be directly imported into OpenLB in order to simulate the thermal conductivity as described in Section 2.2.

Since the entire process for generating the geometry is procedural and parameter driven, multiple geometries can be generated based on the same base geometry. For example, different levels of compression can be applied to the procedurally generated aggregates, allowing direct control over the aggregate size, shape and pore size distributions of the geometries generated. The end result is a set of similarly packed geometries of slightly varying pore and particle sizes. This allows investigation into how small changes in aggregate pore size influence the thermal heat transfer. Furthermore, the effect of aggregate shape and particle size distribution can also be investigated.

\subsection{Thermal conductivity simulation with lattice Boltzmann methods}

As Wang et al. [14] state, LBM is well suited for simulating heat transfer in resolved porous media. Two lattice Boltzmann equations have been combined to solve the conjugate heat transfer through the particle geometry. One lattice is used to solve the diffusion equation for the temperature as described in Section 2.2.1, and a second lattice for radiative transport described in Section 2.2.2. The two lattices are coupled to compute heat flux and effective heat transfer through the porous media. 

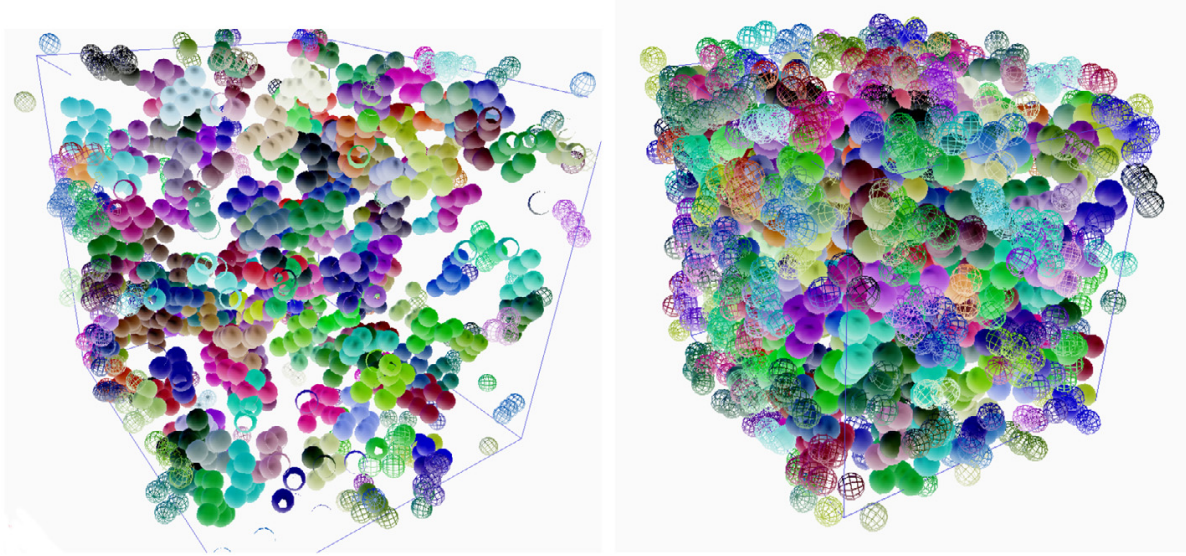

Fig. 1. Geometry generated with YADE before and after compression, aggregates shown in different colors.

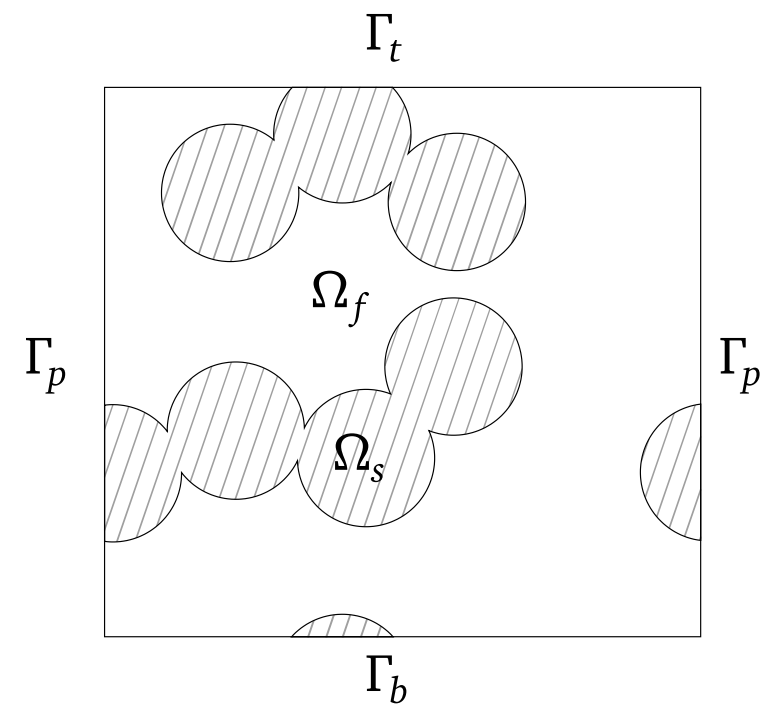

Fig. 2. The two domains for LBM, $\Omega_{s}$ and $\Omega_{f}$ for the solid and fluid respectively, with periodic boundaries $\Gamma_{p}$ and temperature boundaries $\Gamma_{t}$ and $\Gamma_{b}$.

\subsubsection{LBM for energy equation}

To solve the energy equation the domain is composed $\Omega=\Omega_{s} \cup \Omega_{f}$, where $\Omega_{f}$ and $\Omega_{s}$ define the fluid and solid domains as shown in Fig. 2. Following Wang's method for conjugate heat transfer [23] we solve two diffusion equations for the temperature $T$

$$
\begin{aligned}
& \rho_{f} c_{p, f} \frac{\partial T}{\partial t}=\lambda_{f} \nabla^{2} T \text { in } \Omega_{f}, \\
& \rho_{s} c_{p, s} \frac{\partial T}{\partial t}=\lambda_{s} \nabla^{2} T \text { in } \Omega_{s},
\end{aligned}
$$

where $\rho$ is the density and the indices $f$ and $s$ denote the fluid (air) and solid material (silica), respectively, $t$ is the discrete time and $\lambda$ the thermal conductivity.

The diffusion equation can be solved by means of the LBM through

$$
f_{i}(x+\Delta x, t+\Delta t)=f_{i}-\frac{1}{\tau}\left(f_{i}-f_{i}^{e q}\right),
$$

where $f_{i}$ denote the discrete probability function in the discrete direction $i$, at discrete space coordinate $x$ and discrete time $t$. $\Delta x$ and $\Delta t$ denote the grid spacing and time step width, respectively, and $\tau$ denotes the relaxation time. The equilibrium 


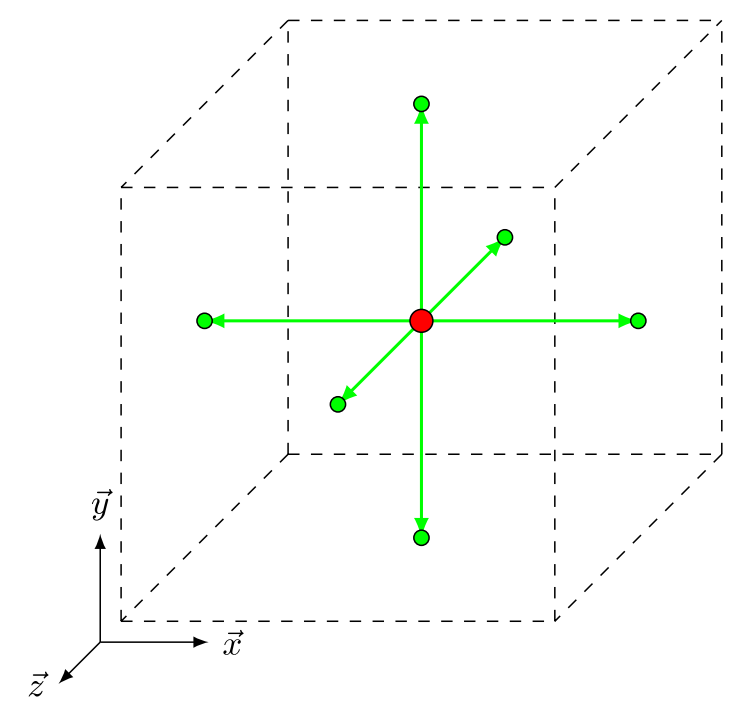

Fig. 3. Schematic representation of the speed directions according to D3Q7 [25].

function $f_{i}^{e q}$ is given according to e.g. [24].

$$
f_{i}^{e q}=w_{i} T(x, t),
$$

wherein $w_{i}$ are the discrete lattice direction's weights and $T(x, t)$ is the local temperature. In this work, we use a three dimensional velocity set with seven discrete directions, referenced as D3Q7 and shown in Fig. 3, with the discrete weights

$$
w_{i}= \begin{cases}\frac{2}{8}, & \text { red direction } \\ \frac{1}{8}, & \text { green directions. }\end{cases}
$$

The local temperature $T(x, t)$ and heat flux $q(x, t)$ are calculated from the distribution function's moments by

$$
T(x, t)=\sum_{i} f_{i}(x, t) \text { and } q(x, t)=\frac{\tau-\frac{1}{2}}{\tau} \sum_{i=0}^{6} c_{i} f_{i}(x, t)
$$

with the discrete velocities $c_{i}$.

Through a Chapman-Enskog expansion [24], the relation between the relaxation time and the thermal diffusivity $\alpha=\frac{\lambda}{\rho c_{p}}$ is

$$
\tau=\frac{\alpha}{c_{s}^{2}}+\frac{1}{2}
$$

with $c_{s}=\frac{1}{\sqrt{4}}$ denoting the lattice speed of sound.

This diffusion equation for the energy distribution is solved for the solid and fluid material on a single lattice with respect to different temperature conductivities through space variant relaxation times $\left.\tau\right|_{\Omega_{s}}=\tau_{s}$ and $\left.\tau\right|_{\Omega_{f}}=\tau_{f}$. As mentioned by Wang et al. [14], this approach is valid for $\rho_{\text {solid }} \cdot c_{p, \text { solid }}=\rho_{\text {fluid }} \cdot c_{p, \text { fluid }}$. To fulfill this condition, we choose the fluid's density in lattice units $\rho_{f}^{*}=1$ and the solid's density in lattice units $\rho_{s}^{*}=\rho_{f}^{*} \frac{c_{p, f}}{c_{p, s}}$.

\subsection{2. $L B M$ for radiation transfer equation}

Mink et al. [26] have shown to solve the P1-approximation of the radiative transfer equation (RTE) by means of LBM by introducing a mesoscopic sink term. Based on the assumptions related to the P1-approximation - homogeneous participating media, spatially constant scattering and absorption parameter $\sigma_{s}$ and $\sigma_{a}$ - the RTE can be transformed into the diffusionreaction equation for the light intensity density $\phi$

$$
\frac{1}{c} \frac{\partial \phi}{\partial t}=\frac{1}{3\left(\sigma_{a}+\sigma_{s}\right)} \nabla^{2} \phi-\sigma_{a} \phi .
$$

Here, $c$ denotes the speed of light and the artificial diffusion coefficient $D$ is defined by

$$
D\left(\sigma_{a}, \sigma_{s}\right)=\frac{1}{3\left(\sigma_{a}+\sigma_{s}\right)} .
$$


Mink proposes the lattice Boltzmann equation

$$
g_{i}(x+\Delta x, t+\Delta t)=g_{i}-\frac{1}{\tau_{g}}\left(g_{i}-g_{i}^{e q}\right)-\frac{3 \sigma_{a}\left(\sigma_{a}+\sigma_{s}\right)}{8} g_{i},
$$

with the discrete probability function $g_{i}$, the equilibrium function $g_{i}^{e q}=w_{i} \phi$ and the relaxation time $\tau_{g}=1$.

The spatial light intensity is given by the distribution function's zeroth moment

$$
\phi(x)=\sum_{i} g_{i}
$$

The boundary radiation intensities are chosen following the Stephan Boltzmann law [27] according to the local lattice temperatures by

$$
\phi=\left(\frac{T_{(x, t)}}{T_{h o t}}\right)^{4} .
$$

\subsubsection{Effective lambda}

Heat transfer through a VIP is composed of the heat transfer through gas $\lambda_{G}$ (convection), through solid $\lambda_{S}$ (conduction), through radiation $\lambda_{R}$ and a coupling term $\lambda_{C}$. The sum of these terms provides the total effective heat transfer

$$
\lambda_{\text {eff }}=\lambda_{G}+\lambda_{S}+\lambda_{R}+\lambda_{C} . \quad \text { in } \mathrm{W} / \mathrm{mK} \text {. }
$$

It should be noted that heat conductivity due to the coupling term is negligible due to the relatively large mean free path of the molecules [7]. The coupling term arises from the increased heat transport bridging between neighboring particles or fibers on the micro-scale and increases as the heat conductivity of the gas and solid increases.

The effective heat conductivity $\lambda_{\text {eff }}$ though the resolved packing is calculated by

$$
\lambda_{\text {eff }}=\frac{q_{\text {eff }} L}{\Delta T}
$$

where $L$ is the cell length, $\Delta T$ is the temperature difference between the upper $\left(\Gamma_{t}\right)$ and lower boundary $\left(\Gamma_{b}\right)$ and the effective heat flux $q_{\text {eff }}$ is given by

$$
q_{\mathrm{eff}}=q_{s}+q_{f}+q_{r} \text {. }
$$

The heat fluxes for the solid $q_{s}$ and for the fluid $q_{f}$ are calculated by the temperature distribution's first momentum in (6). The heat flux due to radiation $q_{r}$ is calculated by

$$
q_{r}=\sigma_{a}\left(4 \sigma_{b} T^{4}-\phi\right),
$$

where $\sigma_{b}$ is the Stephan-Boltzmann constant.

The particle packing's effective thermal conductivity is examined at the bottom layer of the volume in the simulation. The steady state is assumed to be reached, when the calculated effective thermal conductivity does not change more than $0.01 \%$ over the latest 10,000 time steps.

\subsection{Simulation variables}

At low pressures, the heat transfer through a gas (convection) is given by

$$
\lambda_{G}\left(p_{G}\right)=\frac{\lambda_{G 0}}{(1+2 \beta \cdot K n)} \quad \text { in } \mathrm{W} / \mathrm{mK}
$$

where $\beta$ is a weighting factor describing the efficiency of energy transfer from the gas molecules to the solid boundaries and $\lambda_{G O}$ is the effective heat transfer in continuum $\left(26.606 \cdot 10^{-3} \mathrm{~W} / \mathrm{mK}\right.$ [27]). Commonly used $\beta$ values for air and water vapor are 1.63 and 1.5 respectively.

As the pressure inside the VIP decreases, the heat transfer through the fluid decreases inversely proportional to the Knudsen number in (18) [7,27].

$$
K n=\frac{l_{f}}{\Phi}
$$

where $\Phi$ is defined in this work as the pore size of the silica geometry and $l_{f}$ is the mean free path given by

$$
l_{f}=\frac{1}{\sqrt{2} \pi \sigma_{G}^{2} n} \quad \text { in } \mathrm{m} .
$$

n being the number of molecules per volume $\frac{N}{V}\left[\mathrm{~m}^{-3}\right], \sigma_{G}$ the molecular diameter (air): $\sigma_{G}=\left(\sqrt{2} \pi l_{f} n\right)^{-1}=3.7 \cdot 10^{-10} \mathrm{~m}$.

Using (19) and (18), a pressure of $600 \mathrm{mBar}$ and a pore size(characteristic length) of $200 \mathrm{~nm}$ result in a Knudsen number of $0.5 .100 \mathrm{mBar}$ and $200 \mathrm{~nm}: \mathrm{Kn}=3500 \mathrm{mBar}$ and $100 \mathrm{~nm}: \mathrm{Kn}=1.2$. Therefore, the range of Knudsen numbers of interest 


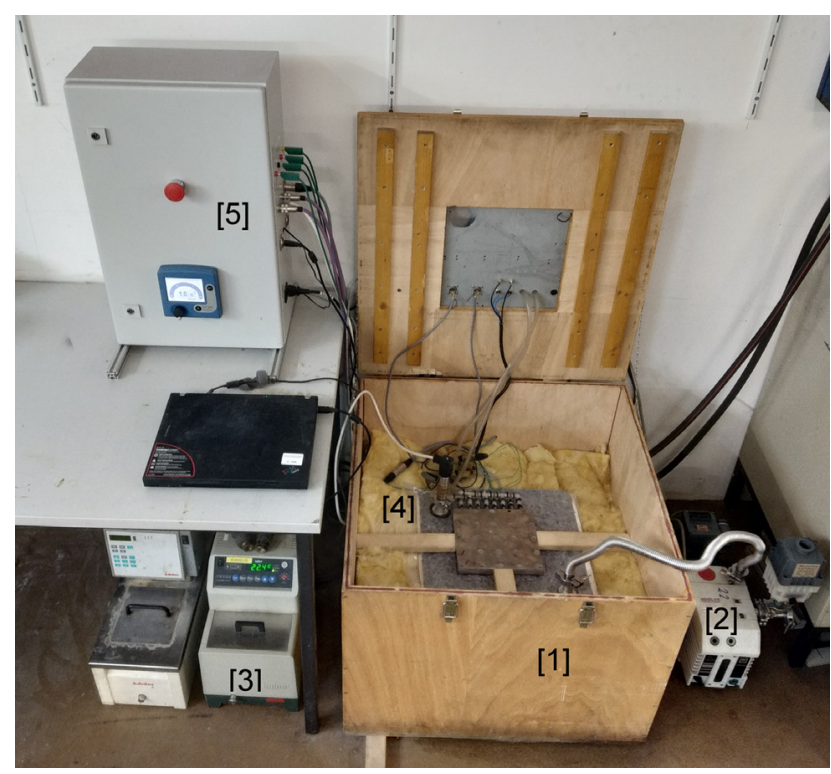

Fig. 4. Thermal conductivity measurement system composed of: [1] insulated enclosure, [2] vacuum pump, [3] thermostat, [4] pressure sensor, [5] measurement electronics and laptop.

is $10^{-1} \leq K n \leq 10^{1}$ or the transition regime. However, free convection can be safely neglected when the pore size is smaller than $3 \mathrm{~mm}$ [20]. An initial estimate for the average pore size $\delta$ is given by (20) from the VDI Heat Atlas [27]. Where $d$ is the average particle diameter and $\Pi$ the porosity of the silica.

$$
\delta=(2 . / 3) \cdot d \cdot \Pi /(1-\Pi) \quad \text { in } \mathrm{m} .
$$

From the ideal gas law, the density of the air, $\rho_{f}$, can be calculated by

$$
\rho_{f}=\frac{p_{G}}{\left(R_{S} \cdot \bar{T}\right)} \quad \text { in } \mathrm{kg} / \mathrm{m}^{3}
$$

where $p_{G}$ is the system pressure, $R_{S}$ the specific gas constant (287.058 J/(kg K) [27]) and the average system temperature $\bar{T}$ is $305.65 \mathrm{~K}$.

The absorption coefficient $\sigma_{a}$ is approximated as

$$
\sigma_{a}=9.2693 \cdot 10^{-3} \cdot \log (P)^{2}+1.3437 \cdot 10^{-1} \cdot \log (P)+4.7933 \cdot 10^{-1},
$$

interpolating from Alberti et al. [28] and Farag [29]. The scattering coefficient $\sigma_{s}$ is estimated as 0.1 for the infrared regime [30]. The heat capacity, $c_{p, s}$, and the density, $\rho_{s}$, of the silica we measured to be $800 \mathrm{~J} / \mathrm{kg} \mathrm{K} \mathrm{and} 238.7 \mathrm{~kg} / \mathrm{m}^{3}$ respectively.

\subsection{Measurements}

In order to measure the thermal conductivity of the VIPs, a measurement system was constructed. The measurement system is composed of a wooden box filled with mineral wool. The VIP is placed between two heating plates underneath and a cooling plate on-top as shown in Fig. 5. The panel has two openings, one connected to a vacuum pump through a solenoid valve, and the second to a Pirani pressure sensor which in combination are used to regulate the pressure in the panel as shown in Figs. 4 and 5. The pump is able to achieve a pressure of $10^{-4} \mathrm{mBar}$.

To measure the thermal conductivity of a panel, the cooling plate is held at a constant temperature of $20^{\circ} \mathrm{C}$ while the top hot plate maintains $40^{\circ} \mathrm{C}$. A second hot plate, placed underneath the first, is used to hinder heat loses through the bottom of the measurement system. The power required to maintain the hot plate temperature is recorded. Once the result reaches a stable level for $750 \mathrm{~s}$, the pressure in the panel is reduced and a new measurement begins. The effective thermal conductivity $\lambda$ is measured according to

$$
\lambda=\frac{\left(\dot{Q}_{M}-\dot{Q}_{L}\right) \cdot d_{V I P}}{A \cdot\left(\bar{T}_{\text {cold }}-\bar{T}_{\text {hot }}\right)} \quad \text { in } \mathrm{W} / \mathrm{mK} .
$$

where $\dot{Q}_{M}$ is the measured power needed to maintain the top hot plate at $313,15 \mathrm{~K}\left(40{ }^{\circ} \mathrm{C}\right), \dot{Q}_{L}$ the measured power losses, $d_{V I P}$ the thickness of the panel, $A$ the area of the panel being measured, $\bar{T}_{\text {cold }}$ is $293,15 \mathrm{~K}\left(20^{\circ} \mathrm{C}\right)$ and $\bar{T}_{\text {hot }}$ is $313,15 \mathrm{~K}\left(40{ }^{\circ} \mathrm{C}\right)$. 


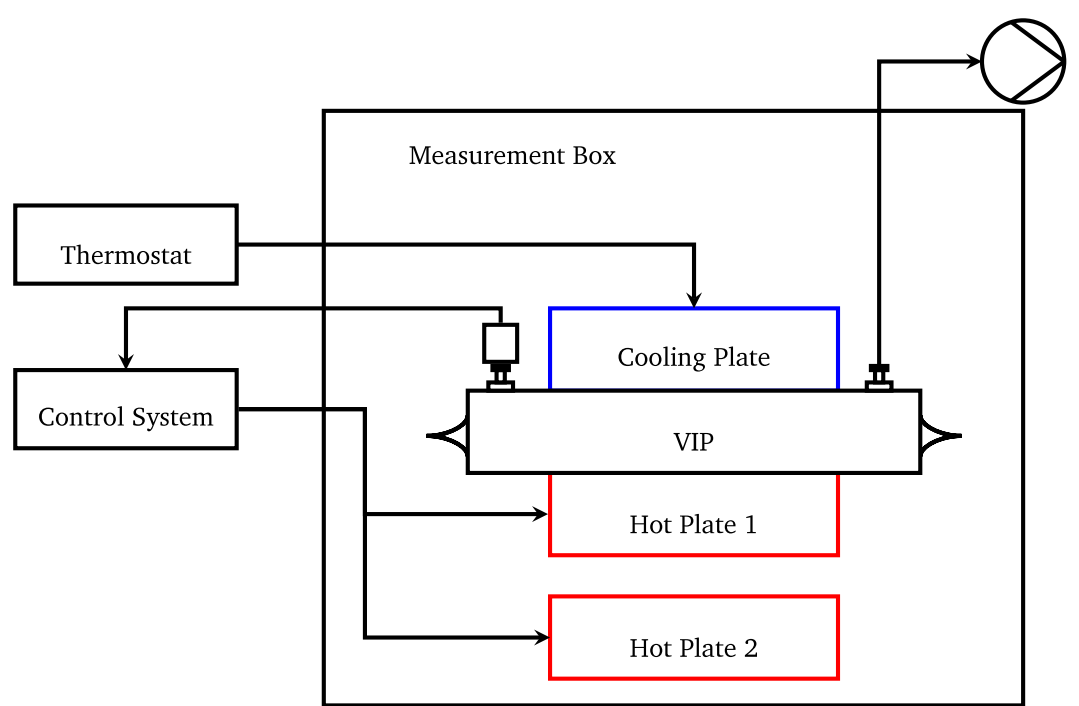

Fig. 5. Schematic diagram of measurement system. The power required to maintain the top hot plate at $40{ }^{\circ} \mathrm{C}$ is proportional to the effective thermal conductivity.

Table 2

Measurement uncertainty for temperature sensors.

\begin{tabular}{lll}
\hline & & Error \\
\hline Thermocouple & PT100 & $\pm 0.270 \mathrm{~K}$ \\
Transducer & MU-PT100-I420 & $0.100 \%$ \\
NIDAQ & USB.6008 & $0.153 \%$ \\
\hline & Total & $0.200 \%$ \\
\hline
\end{tabular}

Table 3

Measurement uncertainty for power sensor.

\begin{tabular}{lll}
\hline & & Error \\
\hline Measure power & Shunt resistor & $1.000 \%$ \\
NIDAQ & USB.6008 & $0.147 \%$ \\
\hline & Total & $1.010 \%$ \\
\hline
\end{tabular}

To determine undesired heat flux to the environment or over the foil surrounding the core material, a well known standard material needs to be measured before every measurement. For this purpose a standard polystyrene board from NIST (National Institute of Standards and Technology) is used. To calculate thermal conductivity as a function of temperature and density the following equation from Zarr et al. [31] holds for the standard material.

$$
\lambda_{\text {standard }}=0.00111-0.0000424 \cdot \rho+0.000115 \cdot T \pm 1.5 \%
$$

To figure out heat losses the test plate needs to be measured in exactly the same way as the sample will be (same foil, same size, same temperatures of cooling and heating plate). The expected heat flux can be calculated with

$$
\dot{Q}_{\text {exp }}=\frac{\lambda_{\text {standard }}}{d} \cdot A \cdot \Delta T \text {. }
$$

The heat losses into the environment and over the foil can now be determined by subtracting the expected heat flux $\dot{Q}_{\text {exp }}$ from the measured one $\left(\dot{Q}_{\text {loss }}=\dot{Q}_{\text {measured }}-\dot{Q}_{\text {exp }}\right)$.

This value can now be used as an offset for the measurement.

\subsection{Measurement uncertainty}

Calculating the propagation of uncertainty using Tables 2 and 3, together with the uncertainty for the thickness and area of the VIP, $5 \%$ and $1 \%$ respectively, in (23), a total uncertainty of $8.73 \%$ for the thermal conductivity measurement is obtained.

Two pressure sensors are used, a Pirani sensor and a Siemens SITRANS P200 capacitive sensor to measure at low and high pressures respectively. The capacitive sensor has a maximum uncertainty of $0.5 \%$, whereas the Pirani sensor is used between 0.01 and 10 mbar and has a maximum uncertainty of $15 \%$. 


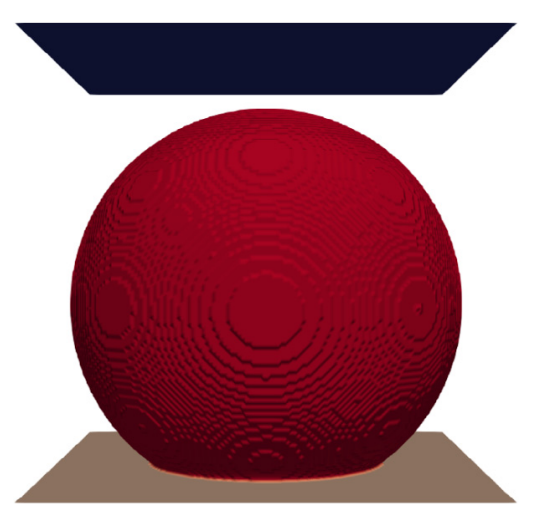

Fig. 6. Simplified geometry used to calculate experimental order of convergence. Top and bottom boundaries are held at constant temperature and the other 4 boundaries are periodic.

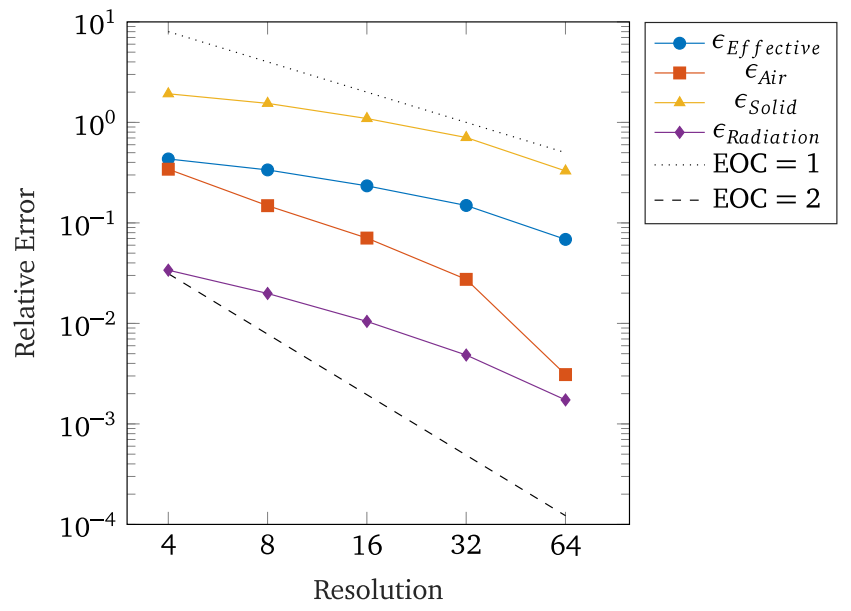

Fig. 7. Relative error as a function of simulation resolution.

\section{Results}

Using the proposed method, several simulation setups were generated to evaluate the reliability and accuracy of the approach. The first study presented is a grid independence study used to confirm convergence of the model. Next, the results of varying air pressure are presented and compared to reported literature values. Lastly the effect of compressing the geometry on the thermal conductivity is presented.

\subsection{Grid independence}

To confirm grid independence, a simplified geometry, shown in Fig. 6, considering a single sphere between two plates, is used to evaluate the effective thermal conductivity. The top boundary $\left(\Gamma_{t}\right)$ is held at a constant temperature of $306.15 \mathrm{~K}$, and the bottom boundary $\left(\Gamma_{b}\right) 305.15 \mathrm{~K}$. The other 4 boundaries are periodic. The radiation intensity is calculated using the local lattice temperature and (12). The resolution of the simulation, $\mathrm{N}$, is defined as the number of grid cells used to resolve the diameter of the sphere. $\mathrm{N}$ is increased with the following steps $4,8,16,32,64$ with diffusive scaling and $\tau_{f}=0.9$. In lieu of an analytical solution a simulation with resolution 128 is used to calculate the relative error by

$$
\epsilon_{\phi}(N)=\frac{\lambda_{\phi}(N)-\lambda_{\phi}\left(N_{\max }\right)}{\lambda_{\phi}\left(N_{\max }\right)}
$$

where $\phi$ is the effective thermal conductivity of air, solid or radiation and $\epsilon$ the relative error. The relative error versus the system resolution is shown in Fig. 7. Thermal conductivity through air and due to radiation have a superlinear experimental order of convergence. The thermal conductivity through the solid is sublinear due to the abundance of curved boundaries. 


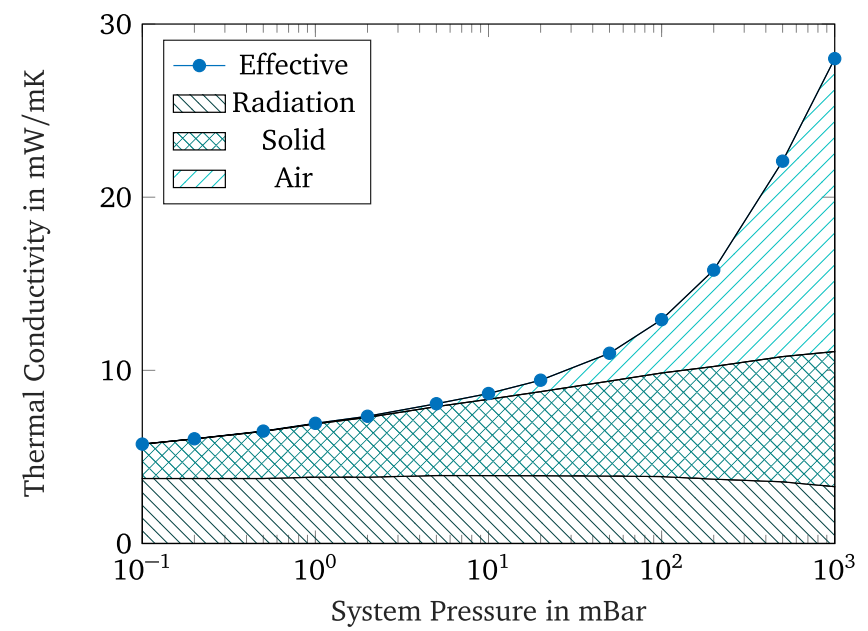

Fig. 8. Heat conductivity breakdown due to air, radiation and through the solid material.

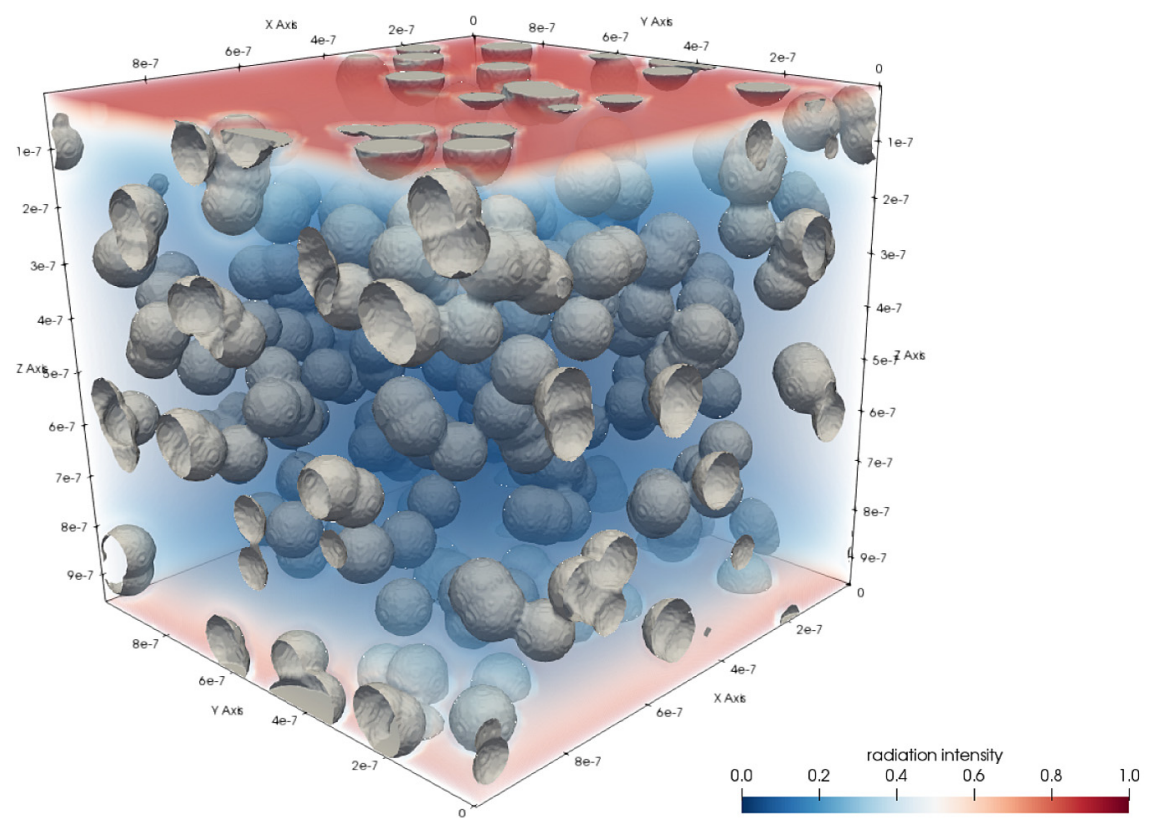

Fig. 9. Radiation distribution through periodic packing of silica aggregates.

\subsection{Effective thermal conductivity as a function of pressure}

To evaluate the thermal conductivity of our silica geometry, the top boundary $\left(\Gamma_{t}\right)$ is held at a constant temperature of $306.15 \mathrm{~K}$, and the bottom boundary $\left(\Gamma_{b}\right)$ at $305.15 \mathrm{~K}$. The other 4 boundaries are periodic. The radiation intensity is calculated using the local lattice temperature and (12). The resolution of the simulation, $\mathrm{N}$, is 16. Fig. 8 shows the thermal conductivity breakdown as a function of pressure. The total thermal conductivity is composed of the thermal conductivity due to the air, radiation and solid. As the system pressure decreases, the thermal conductivity of the air clearly decreases. Below 50 mBar the thermal conductivity of air ceases to have a significant effect. The radiation and temperature distributions in the geometry can be seen in Figs. 9 and 10 respectively.

The thermal transfer due to radiation remains relatively constant as the system pressure decreases. At higher pressures, the number of air particles also increases and thus absorption of radiation increases and the heat flux due to radiation decreases slightly.

Likewise, the thermal conductivity through the solid decreases as the system pressure decreases. While this should not be the case at very low pressures, this is essentially the coupling effect seen between particles. This is due to the air providing a second parallel path for the heat, increasing therefore the contribution through the solid. As the pore size, and therefore 


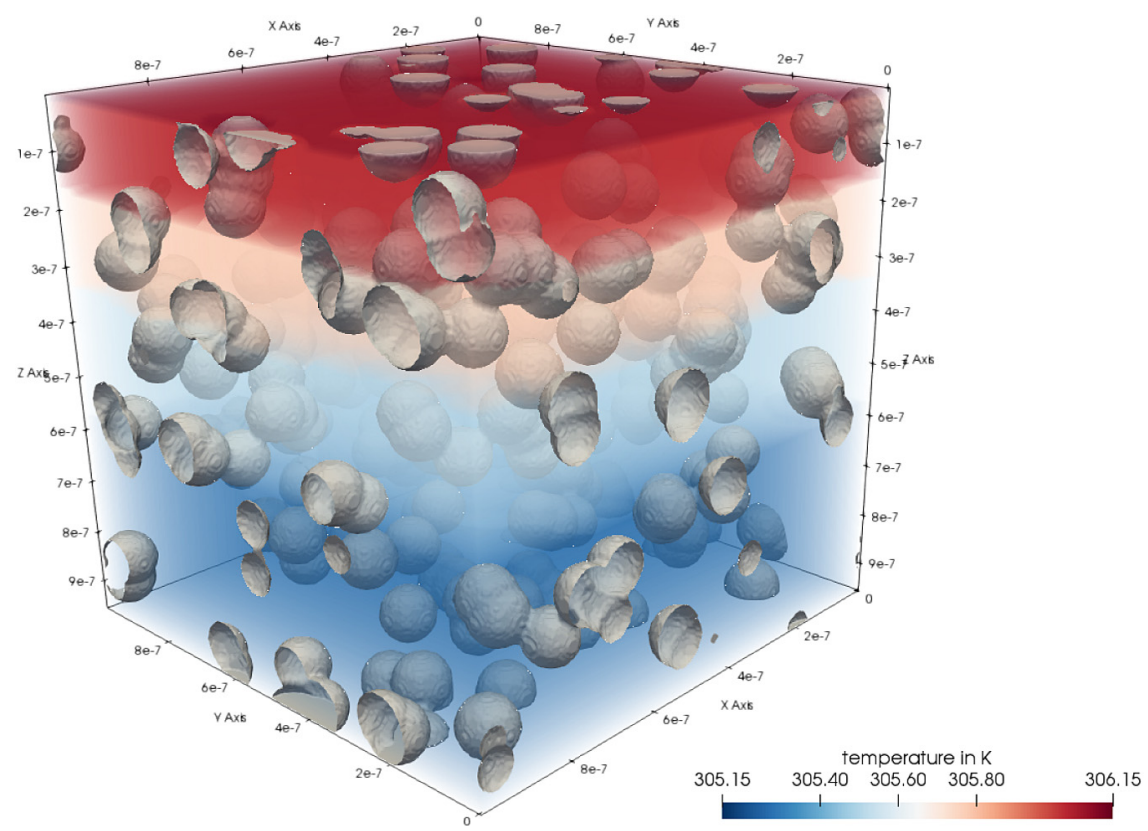

Fig. 10. Temperature distribution through periodic packing of silica aggregates.

mean free path, of the system is defined globally, these 'short cuts' between particles exist even at low pressure. This is a shortcoming of using a globally defined pore size. The solution would be to calculate the pore size for every lattice unit and therefore define a thermal conductivity per lattice. This would significantly reduce the thermal conductivity at lower pressures.

\subsection{Comparing with literature and measured values}

Fig. 11 shows a comparison between the simulations results and literature values provided by Fricke et al. [7] for heat conductivity of silica at various pressures. Similar to the previous test case, the top boundary $\left(\Gamma_{t}\right)$ is held at a constant temperature of $306.15 \mathrm{~K}$, and the bottom boundary $\left(\Gamma_{b}\right)$ at $305.15 \mathrm{~K}$. The other 4 boundaries are periodic. The radiation intensity is calculated using the local lattice temperature and (12). The resolution of the simulation, $\mathrm{N}$, is 16.

Literature values show that a constant thermal conductivity is reached below $10 \mathrm{mBar}$. Whereas the thermal conductivity in the simulation continues to decrease slowly. As previously mentioned this is due to the solid conductivity and a global pore size and mean free path. In order to obtain improved accuracy at low air pressures, the local pore size of each lattice unit will be calculated and therefore define a thermal conductivity per lattice. The relative error between the simulated and reference values is $6.12 \%$.

The simulations are also compared to measured thermal conductivities of VIP panels compressed under various pressures. The VIP panels compressed with 25 and 30 bar closely follow the simulations with compression levels 0 and 2 respectively, both with a relative error of $3.7 \%$.

\subsection{Effect of compression on thermal conductivity}

In order to investigate the effect of different compression forces on the thermal conductivity of the VIPs, several panels were compressed and their thermal conductivity measured. The results of compressing the panels with 15, 20, 25 and 30 Bar are shown in Fig. 12a. As the compression force increases, the thermal conductivity through the panel decreases. One explanation for this phenomenon is that the pore size decreases with increasing compression force. Therefore, as discussed in Section 2.3 the Knudsen number decreases and as a result the thermal conductivity through the air also decreases.

In order to see if a similar effect would be observed in the simulation, various geometries were generated with varying degrees of compression. The porosity and average pore size was calculated for each geometry and then used to simulate the heat transfer through the generated geometry in OpenLB. The results are shown in Fig. 12b, where the compression level indicates the number of iterations applied to compress the particle geometry. A similar tendency to that which was observed in the measurements is revealed; the thermal conductivity decreases as the particle geometry is compressed. However, further work is needed to link the specific compression force to simulated compression levels in YADE. $3.5 \mathrm{~mW} / \mathrm{mK}$ is added to correct for thermal bridging artifacts and power losses in the measurement. 


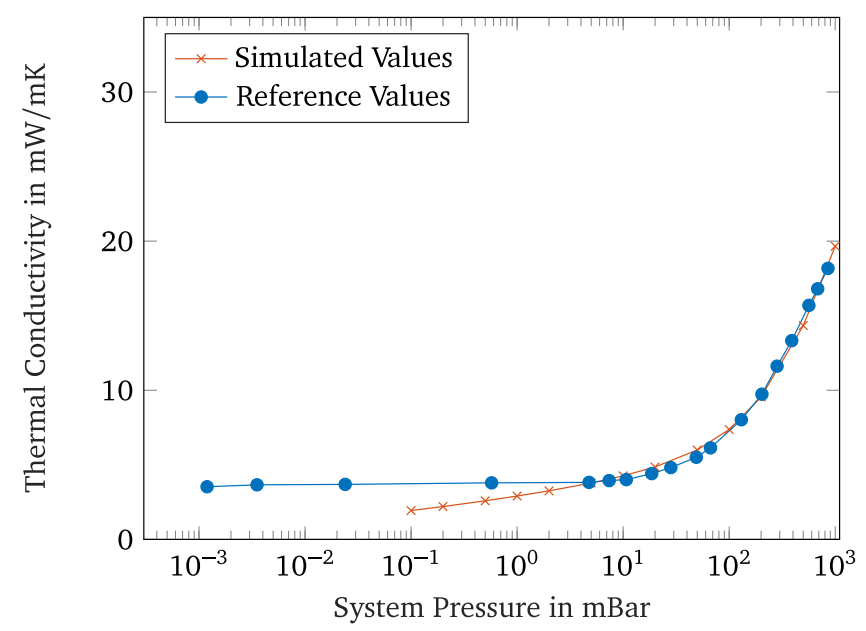

Fig. 11. Comparison of effective thermal conductivity to reference values [7]. Simulated effective thermal conductivity decreases at low pressures due to solid conductivity being influenced by the globally defined mean free path.

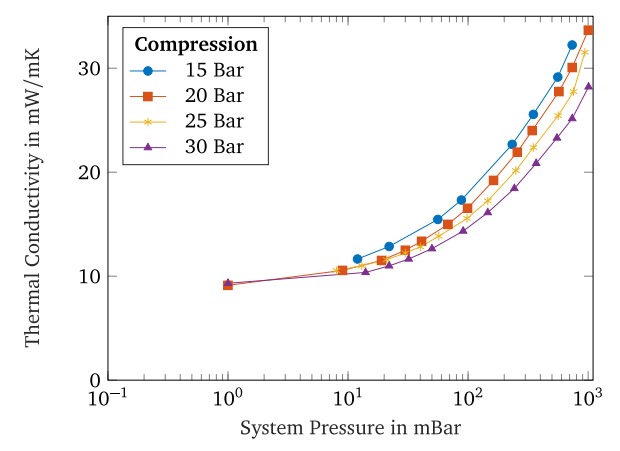

(a) Measured values

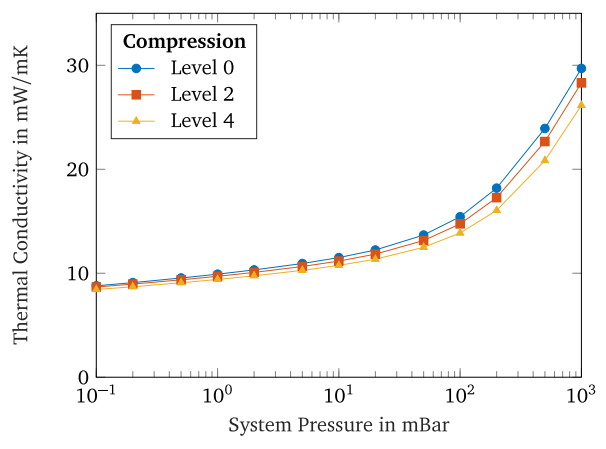

(b) Simulated values

Fig. 12. (A) Measurement of the effect different compression forces on the thermal conductivity of the VIP. (B) Simulation results of the effect of different compression forces on the thermal conductivity of the VIP $(3.5 \mathrm{~mW} / \mathrm{mK}$ is added to correct for thermal bridging artifacts in the measurement).

\section{Conclusion}

The goal of this work was to generate a holistic method to simulate the effective heat transfer through vacuum insulation panels using procedurally generated 3D geometry of silica aggregates. This was achieved through combination of two open source software packages, YADE DEM and OpenLB. Comparisons were provided that show simulation results closely match measured effective heat transfers of real VIPs. We are now able to directly control and tune particle packing characteristics such as aggregate size, shape and pore size distributions and study their influence directly on the heat transfer through VIPs in the form of conduction and radiation independently. The simulations are performed over a range of internal pressures and the geometry is compressed over varying degrees. The simulation results are compared to both reference values and measured values, enhancing the credibility of these results. Work has already begun developing new packing geometries which will result in cost reduction and increased lifespan of VIPs by increasing the minimum pressure required for low effective thermal conductivity.

\section{Acknowledgments}

The authors would like to thank the German Central Innovation Programme for SMEs, Germany for funding this project (ZF4013908CL5). This work was performed on the computational resource ForHLR II funded by the Ministry of Science, Research and the Arts Baden-Württemberg, Germany and DFG ("Deutsche Forschungsgemeinschaft"), Germany. 


\section{References}

[1] A. Capozzoli, S. Fantucci, F. Favoino, M. Perino, Vacuum insulation panels: Analysis of the thermal performance of both single panel and multilayer boards, Energies 8 (4) (2015) 2528-2547, http://dx.doi.org/10.3390/en8042528.

[2] S.E. Kalnaes, B.P. Jelle, Vacuum insulation panel products: A state-of-the-art review and future research pathways, Appl. Energy 116 (7465) (2014) 355-375, http://dx.doi.org/10.1016/j.apenergy.2013.11.032.

[3] A. Berge, P. Johansson, Literature Review of High Performance Thermal Insulation, Department of Civil and Environmental Engineering, Chalmers University of Technology, Goteborg, 2012, p. 40.

[4] F. Enguehard, Multi-scale modeling of radiation heat transfer through nanoporous superinsulating materials, Int. J. Thermophys. 28 (5) (2007) 16931717, http://dx.doi.org/10.1007/s10765-006-0143-1

[5] S. Spagnol, B. Lartigue, A. Trombe, V. Gibiat, Thermal modeling of two-dimensional periodic fractal patterns, an application to nanoporous media, Europhys. Lett. (EPL) 78 (4) (2007) 46005, http://dx.doi.org/10.1209/0295-5075/78/46005.

[6] J.S. Kwon, C.H. Jang, H. Jung, T.H. Song, Effective thermal conductivity of various filling materials for vacuum insulation panels, Int. J. Heat Mass Transfer $52(23-24)(2009)$ 5525-5532, http://dx.doi.org/10.1016/j.ijheatmasstransfer.2009.06.029.

[7] J. Fricke, Vakuum-Isolations-Paneele für Gebäude, ZAE Bayern, 2007, p. 134.

[8] J. Kim, T.-H. Song, Vacuum insulation properties of glass wool and opacified fumed silica under variable pressing load and vacuum level, Int. J. Heat Mass Transfer 64 (2013) 783-791, http://dx.doi.org/10.1016/j.ijheatmasstransfer.2013.05.012.

[9] D. Rochais, G. Domingues, F. Enguehard, Numerical simulation of thermal conduction and diffusion through nanoporous superinsulating materials, in: Proceedings of the 17th European Conference on Thermophysical Properties, 2005.

[10] R. Coquard, D. Baillis, V. Grigorova, F. Enguehard, D. Quenard, P. Levitz, Modelling of the conductive heat transfer through nano-structured porous silica materials, J. Non-Cryst. Solids 363 (2013) 103-115, http://dx.doi.org/10.1016/j.jnoncrysol.2012.11.053.

[11] S. Lallich, F. Enguehard, D. Baillis, Experimental determination and modeling of the radiative properties of silica nanoporous matrices, J. Heat Transfer 131 (8) (2009) 082701, http://dx.doi.org/10.1115/1.3109999.

[12] M. Wang, N. Pan, Modeling and prediction of the effective thermal conductivity of random open-cell porous foams, Int. J. Heat Mass Transfer 51 (5-6) (2008) 1325-1331, http://dx.doi.org/10.1016/j.ijheatmasstransfer.2007.11.031.

[13] P. Ferkl, R. Pokorný, M. Bobák, J. Kosek, Heat transfer in one-dimensional micro- and nano-cellular foams, Chem. Eng. Sci. 97 (2013) 50-58, http://dx.doi.org/10.1016/j.ces.2013.04.018.

[14] M. Wang, J. Wang, N. Pan, S. Chen, Mesoscopic predictions of the effective thermal conductivity for microscale random porous media, Phys. Rev. E 75 (3) (2007) 036702, http://dx.doi.org/10.1103/PhysRevE.75.036702.

[15] A. Kaemmerlen, C. Vo, F. Asllanaj, G. Jeandel, D. Baillis, Radiative properties of extruded polystyrene foams: Predictive model and experimental results, J. Quant. Spectrosc. Radiat. Transfer 111 (6) (2010) 865-877, http://dx.doi.org/10.1016/j.jqsrt.2009.11.018.

[16] Z. Qu, Y. Fu, Y. Liu, L. Zhou, Approach for predicting effective thermal conductivity of aerogel materials through a modified lattice Boltzmann method, Appl. Therm. Eng. 132 (2018) 730-739, http://dx.doi.org/10.1016/j.applthermaleng.2018.01.013.

[17] G. Falcucci, S. Succi, A. Montessori, S. Melchionna, P. Prestininzi, C. Barroo, D.C. Bell, M.M. Biener, J. Biener, B. Zugic, E. Kaxiras, Mapping reactive flow patterns in monolithic nanoporous catalysts, Microfluid. Nanofluid. 20 (7) (2016) 105, http://dx.doi.org/10.1007/s10404-016-1767-5.

[18] V. Krastev, G. Falcucci, Simulating engineering flows through complex porous media via the lattice boltzmann method, Energies 11 (4) (2018) 715 , http://dx.doi.org/10.3390/en11040715.

[19] G. Falcucci, G. Amati, V.K. Krastev, A. Montessori, G.S. Yablonsky, S. Succi, Heterogeneous catalysis in pulsed-flow reactors with nanoporous gold hollow spheres, Chem. Eng. Sci. 166 (2017) 274-282, http://dx.doi.org/10.1016/j.ces.2017.03.037.

[20] F. Govan, D. Greason, J. McAllister (Eds.), Thermal Insulation, Materials, and Systems for Energy Conservation in the '80s, ASTM International, 100 Barr Harbor Drive, PO Box C700, West Conshohocken, PA 19428-2959, 1983. http://dx.doi.org/10.1520/STP789-EB.

[21] J. Fietz, M.J. Krause, C. Schulz, P. Sanders, V. Heuveline, Optimized Hybrid Parallel Lattice Boltzmann Fluid Flow Simulations on Complex Geometries, in: Lecture Notes in Computer Science (including subseries Lecture Notes in Artificial Intelligence and Lecture Notes in Bioinformatics), vol. 7484 LNCS, 2012, pp. 818-829, http://dx.doi.org/10.1007/978-3-642-32820-6_81.

[22] V. Šmilauer, B. Chareyre, J. Duriez, A. Eulitz, A. Gladky, N. Guo, C. Jakob, J. Kozicki, Using and Programming, Yade Documentation, 2015. http: //dx.doi.org/10.5281/zenodo.34043.

[23] J. Wang, M. Wang, Z. Li, A lattice Boltzmann algorithm for fluidsolid conjugate heat transfer, Int. J. Therm. Sci. 46 (3) (2006) 228-234, http: //dx.doi.org/10.1016/j.ijthermalsci.2006.04.012.

[24] A.A. Mohamad, Lattice Boltzmann Method, Springer London, London, 2011, http://dx.doi.org/10.1007/978-0-85729-455-5.

[25] M. Gaedtke, S. Wachter, M. Rädle, H. Nirschl, M.J. Krause, Application of a Lattice Boltzmann method combined with a smagorinsky turbulence model to spatially resolved heat flux inside a refrigerated vehicle, Comput. Math. Appl. (2018) http://dx.doi.org/10.1016/j.camwa.2018.08.018.

[26] A. Mink, G. Thäter, H. Nirschl, M.J. Krause, A 3D Lattice Boltzmann method for light simulation in participating media, J. Comput. Sci. 17 (2016) 431-437, http://dx.doi.org/10.1016/j.jocs.2016.03.014.

[27] VDI e.V., VDI-Wärmeatlas, Springer Berlin Heidelberg, Berlin, Heidelberg, 2013. http://dx.doi.org/10.1007/978-3-642-19981-3.

[28] M. Alberti, R. Weber, M. Mancini, Re-creating Hottel's emissivity charts for water vapor and extending them to 40 bar pressure using HITEMP-2010 data base, Combust. Flame 169 (2016) 141-153, http://dx.doi.org/10.1016/j.combustflame.2016.04.013.

[29] I.H. Farag, Radiative Heat Transmission from Non-luminous Gases. Computational Study of the Emissivities of Water Vapor and Carbon Dioxide (Ph.D. thesis), Massachusetts Institute of Technology, 1978.

[30] R.W. Fenn, S.A. Clough, W.O. Gallery, R.E. Good, F.X. Kneizys, J. Mill, L. Rothman, E. Shettle, F. Volz, Optical and infrared properties of the atmosphere, in: Handbook of Geophysics and the Space Environment, 1985, pp. 1-80.

[31] R.R. Zarr, A.L. Pintar, Standard Reference Materials: SRM 1453, Expanded Polystyrene Board, for Thermal Conductivity from 281 K to 313 K, Tech. Rep., National Institute of Standards and Technology, Gaithersburg, MD, 2012, http://dx.doi.org/10.6028/NIST.SP.260-175. 
Karlsruher Institut für Technologie

\section{Repository KITopen}

Dies ist ein Postprint/begutachtetes Manuskript.

\section{Empfohlene Zitierung:}

Ross-Jones, J.; Gaedtke, M.; Sonnick, S.; Rädle, M.; Nirschl, H.; Krause, M. J. Conjugate heat transfer through nano scale porous media to optimize vacuum insulation panels with lattice Boltzmann methods. 2019. Computers and mathematics with applications, 77 doi: $\underline{10.5445 / I R / 1000097090}$

Zitierung der Originalveröffentlichung:

Ross-Jones, J.; Gaedtke, M.; Sonnick, S.; Rädle, M.; Nirschl, H.; Krause, M. J. Conjugate heat transfer through nano scale porous media to optimize vacuum insulation panels with lattice Boltzmann methods.

2019. Computers and mathematics with applications, 77 (1), 209-221. doi:10.1016/j.camwa.2018.09.023 